\title{
TRANSITIVITIES IN PROJEGTIVE PLANES
}

\author{
T. G. OSTROM
}

Introduction. A perspectivity in a projective plane is a collineation which leaves some line pointwise fixed. Baer (4) has considered the coordinatization of planes which admit certain groups of perspectivities. André $(\mathbf{1} ; \mathbf{2} ; \mathbf{3})$ has made an extensive study of the Veblen-Wedderburn plane in terms of its perspectivities. The author has shown (7) that finite doubly transitive planes are Desarguesian if the number of points on a line is $n+1$ where $n$ is an odd non-square.

In Part 1, we consider certain products of perspectivities. Our main results are certain uniqueness theorems for the group of permutations of points on a line induced by perspectivities.

In Part 2 , we consider the coordinatization of planes admitting two $p-L$ transitive groups. (See definition in Part 1.) In various cases, depending upon the kinds of perspectivities and the relative location of the centers and axes, we obtain planes which can be coordinatized by various types of algebraic systems.

In Part 3, we consider the exceptional cases not taken care of in the author's paper on double transitivity. We dispose of the case where $n$ is an odd power of 2 . For all values of $n$, we show that transitivity on quadrangles implies that every quadrangle generates a Desarguesian subplane.

In Part 4, we show that every finite plane contains Desarguesian configurations.

\section{Uniqueness theorems on perspectivities.}

Definition. A collineation which leaves fixed every line through some point $p$ (called the center) and every point on some line $L$ (called the axis) is a perspectivity.

We shall make use of the well-known fact that the above definition is redundant in the sense that if every line through $p$ is fixed then there is some line $L$ such that every point on $L$ is fixed (4).

Definition. A perspectivity in which the center is on the axis will be called an elation. A perspectivity in which the center is not on the axis will be called a homology.

Definition. Let $\Sigma$ be the group of perspectivities with center $p$ and axis $L$. Then, if $\Sigma$ is transitive on the non-fixed points of each line through $p$, the plane is said to be $p-L$ transitive.

Received September 10, 1956. 
LEMMA 1. Let $\rho$ and $\sigma$ be two homologies with the same center $p$, and let the axes of $\rho$ and $\sigma$ intersect in some point $q \neq p$. Let $r$ be any point on the line $p q$ other than $p$ or $q$. Then if $r$ is fixed by $\rho \sigma, \rho \sigma$ is a perspectivity with center $p$ and axis $q r$.

Proof. Since $\rho \sigma$ leaves every line through $p$ fixed, $\rho \sigma$ is a perspectivity with center $p$. Moreover, both $q$ and $r$ are fixed by $\rho \sigma$. For a perspectivity all fixed points other than the center must lie on the axis. Hence $q r$ is the axis of $\rho \sigma$.

THEOREM 1. Let $\rho$ and $\sigma$ be two homologies with the same center $p$, and let the axes of $\rho$ and $\sigma$ intersect in some point $q \neq p$. If some point $r$ (other than $p$ or $q$ ) on the line $p q$ has the same image $r_{1}$ under both $\rho$ and $\sigma$, then $\rho$ and $\sigma$ produce the same permutation of points on the line rq.

Proof. By Lemma 1, $\rho \sigma^{-1}$ leaves every point of $r q$ fixed.

Corollary. Suppose that the plane is $p-L$ transitive, where $p \notin L$. Let $L_{1} \neq L$ be some line through $p$ intersecting $L$ in some point $q \neq p$. Let $\rho$ be a collineation fixing both $p$ and $q$. Let $\Sigma$ be the group of $p-L$ perspectivities, and let $G$ be the induced permutation group on the line $p q$. Then $\rho^{-1} \Sigma \rho$ induces an automorphism of $G$.

Proof. If $r$ and $r_{1}$ are two points of the line $p q$, other than $p$ or $q$, there is exactly one member of $\Sigma$ (and hence of $G$ ) which carries $r$ into $r_{1}(4)$. But $\rho^{-1} \Sigma_{\rho}$ is another transitive group of perspectivities with center $p$ and some axis through $q$. It follows from Theorem 1 that the group $G_{1}$ induced by $\rho^{-1} \Sigma \rho$ is the same as $G$.

LEMMA 2. Let $\rho$ and $\sigma$ be two elations with the same center $p$. Let $r$ be any point $\neq p$. Then if $r$ is fixed by $\rho \sigma, \rho \sigma$ is an elation with center $p$ and axis $r p$.

Proof. Since every line through $p$ is fixed by $\rho \sigma, \rho \sigma$ is a perspectivity with center $p$. Let $L$ be the axis of $\rho$. If the axis of $\rho \sigma$ does not go through $p$, it contains some point $q \neq p$ on $L$. The point $q$ is a fixed point of $\rho$ and $\rho \sigma$, and hence is a fixed point of $\sigma$. But the only fixed points of an elation are those on the axis. Thus $p q=L$ is the axis of $\sigma$. We conclude that the axis of $\rho \sigma$ does go through $p$ and $\rho \sigma$ is an elation. The lemma follows from the fact that the only fixed points of an elation are those on the axis.

THEOREM 2. Let $\rho$ and $\sigma$ be two elations with the same center $p$. If $r \rightarrow r_{1}$ under both $\rho$ and $\sigma$, then $\rho$ and $\sigma$ produce the same permutation of points on pr.

Corollary. Suppose that the plane is $p-L$ transitive, where $p \in L$. Let $L_{1} \neq L$ be some line through $p$. Let $\rho$ be a collineation fixing $p$ and $L_{1}$. Let $\Sigma$ be the group of $p-L$ perspectivities, and let $G$ be the induced permutation group on $L_{1}$. Then $\rho^{-1} \Sigma \rho$ is an automorphism of $G$. 
Theorem 3. Let $\rho$ and $\sigma$ be two elations with the same center $p$ but with different axes $L$ and $M$. Then $\rho$ and $\sigma$ are of the same period.

Proof. Consider the elation $\rho \sigma$. Points on $L$ are permuted in cycles whose length is equal to the period of $\sigma$ and points on $M$ are permuted in cycles whose length is equal to the period of $\rho$. But the lengths of both of these cycles are equal to the period of $\rho \sigma$. (We are here using the fact that the non-fixed points of a perspectivity are all permuted in cycles of the same length. This in turn depends upon the fact that a perspectivity can have no fixed points off the axis, other than the center.)

2. Perspectivities and coordinate systems. In this section, we shall be using Hall's method (5) of coordinatizing a plane, in which the equation of a line is represented by a ternary operation $y=x \cdot m \circ b$. "Addition" and "multiplication," respectively, are defined in terms of the ternary by $x m=x \cdot m \circ 0$ and $x+b=x \cdot 1 \circ b$. It is not, in general, true that $x \cdot m \circ b=x m+b$. If this identity holds, the coordinatization is said to be linear.

We shall depart from our convention of using small letters for points and capital letters for lines to the extent that we shall use " $A$ " to indicate the center of the pencil $x=$ constant, and " $B$ " for the center of the pencil $y=$ constant.

Our purpose in this part is to consider the coordinate systems which arise (with the proper choice of the quadrangle of reference) in various cases in which the plane admits two $p-L$ transitive groups. We shall refer to some of Baer's results on $p-L$ transitivity (4). Baer's method of coordinatization is different from that of Hall; however, they amount to the same thing if $a \cdot 1=a$ in Baer's system or $a \cdot(-1)=-a$ in Hall's system. By and large, the same methods apply in both cases, so we shall apply some of Baer's results to Hall's system without further explanation.

For convenience, we include the definition of the more common coordinatizing algebras, and some theorems already known.

Definition. An algebraic system consisting of two binary operations, addition and multiplication, is called a Veblen-Wedderburn system, or quasifield, if it satisfies the following conditions:

1. Addition is an Abelian group.

2. $(b+c) a=b a+c a$.

3. $a \cdot 0=0 \cdot a=0$.

4. $1 \cdot a=a \cdot 1=a$.

5. $a y=b$ has a unique solution $y$ if $a \neq 0$.

6. $-x b+x a=c$ has a unique solution $x$ if $a \neq b$.

The system is called a nearfield if

7. Multiplication is associative.

If condition 2 is replaced by the other distributive law, $a(b+c)=a b+a c$, with corresponding interchange of right and left multiplication in 5 and 6 , 
we shall call the system a dual Veblen-Wedderburn system or dual nearfield as is appropriate.

Definition. A plane which can be coordinatized by a Veblen-Wedderburn system with $x \cdot m \circ b=x m+b$ is a Veblen-Wedderburn plane.

It is well known that the dual of a Veblen-Wedderburn plane can be coordinatized by a dual Veblen-Wedderburn system.

Definition. A pair of points $p$ and $q$ on the line at infinity of a Veblen-Wedderburn plane is said to be admissible if the plane is $p-L$ transitive for some line $L$ through $q$, where $L \neq L_{\infty}$.

THEOREM 4 (André (3)). There are exactly the following possibilities for the set $Z$ of all admissible pairs of points of a Veblen-Wedderburn plane $\pi$ :

I. $Z$ is empty.

II. $Z$ consists of exactly one pair $(p, p)$. In this case, $\pi$ is a plane over a distributive quasifield, which is not a left alternative field.

III. $Z$ consists of all pairs $(p, p)$ on $L_{\infty}$. In this case, $\pi$ is a plane over a left alternative field which is not a skewfield.

IV. $Z$ consists of exactly two pairs $(p, q)$ and $(q, p)$ with $q \neq p$. In this case, $\pi$ is a plane over a near field which contains more than 9 elements and is not a skewfield.

V. For each $p$ on $L_{\infty}$ there exists one $q \neq p$ such that $(p, q)$ and $(q, p)$ belong to $Z$. In this case, $\pi$ is the plane over the nearfield of order 9 .

VI. $Z$ consists of all pairs of points on $L_{\infty}$. In this case, $\pi$ is Desarguesian.

Theorem 5 (Baer (4)). If the plane is $A-L_{\infty}$ transitive, then (i) addition is a group and (ii) the coordinatization is linear.

Theorem 6 (Pickert (9)). If the plane is $B-(x=0)$ transitive, then (i) multiplication is associative and (ii) the coordinatization is linear.

In each of the remaining theorems of this section, it is to be understood that the plane is $p_{1}-L_{1}$ transitive and also $p_{2}-L_{2}$ transitive. It is also to be understood that $p_{1}$ is different from $p_{2}$ and $L_{1}$ is different from $L_{2}$ unless equality is specifically indicated.

Theorem 7. If $L_{1}=L_{2}=L$ and both $p_{1}$ and $p_{2}$ are on $L$, the plane is a Veblen-Wedderburn plane with $L$ as the line at infinity.

Proof. By (4, Lemma 3.2), the plane is $p-L$ transitive for every point $p$ on $L$. This implies that the plane is a Veblen-Wedderburn plane. See (1).

THEOREM 8. If $p_{1}$ is on $L_{1}$ and $p_{2}$ is the intersection of $L_{1}$ and $L_{2}$, the plane can be coordinatized by a distributive quasifield.

Proof. By the use of the $p_{2}-L_{2}$ transitivity, $p_{1}$ can be carried into any point on $L_{1}$ except $p_{2}$. Hence, by Theorem 7 , we have a Veblen-Wedderburn plane with $L_{1}$ as $L_{\infty}$. Theorem 8 then follows from Theorem 4. 
Theorem 9. If $p_{1}=p_{2}=p, L_{1}$ goes through $p$ and $L_{2}$ does not go through $p$, then the plane can be coordinatized by a nearfield.

Proof. Take $p \equiv A, L_{1} \equiv L_{\infty}$ and $L_{2} \equiv(y=0)$. Then, by Theorem 5 , the coordinatization is linear and addition is a group. Now consider the $A-(y=0)$ perspectivity which carries the point $(0,1)$ into $(0, a)$. Since $B$ is fixed, $y=1 \rightarrow y=a$. Since every line through $A$ is fixed, the $x$ coordinate of every point is unchanged. In particular,

$$
(1,1) \rightarrow(1, a)
$$

Hence $y=x \rightarrow y=x a$. It follows that $(c, c) \rightarrow(c, c a)$ and $y=c \rightarrow y=c a$. Thus, the right multiplications are isomorphic to the group of $A-(y=0)$ perspectivities, and multiplication by non-zero elements is associative. Now, if (1) indicates the point at infinity corresponding to slope 1 and $(a)$ the point at infinity corresponding to slope $a$, we have

$$
(1) \rightarrow(a) \text {; moreover, }(0, b) \rightarrow(0, b a) \text {. }
$$

Hence, $y=x+b \rightarrow y=x a+b a$. But $(c, c+b) \rightarrow(c,(c+b) a)$. The latter point must be on the line $y=x a+b a$. Hence $(c+b) a=c a+b a$.

André (1, Theorem 13) has shown that addition is abelian if all of the other properties of a Veblen-Wedderburn system are satisfied. Properties 3, 4, $\tilde{0}$ and 6 of a Veblen-Wedderburn system follow from the general properties of Hall's ternary and the theorem follows. Baer (4) has given the proof for the dual of this case.

THEOREM 10. If $p_{1}$ is on both $L_{1}$ and $L_{2}$ and $p_{2}$ is on $L_{2}$ and if we take $p_{1} \equiv A_{1}$, $p_{2} \equiv B, L_{2} \equiv(x=0)$, we have (i) the coordinatization is linear (ii) addition forms a group and (iii) multiplication is associative.

Proof. Follows from Theorems 5 and 6.

THEOREM 11. If $p_{1}$ and $p_{2}$ are on $L_{1}$, but $L_{2}$ does not go through $p_{1}$ or $p_{2}$, then the plane can be coordinatized by a nearfield.

Proof. Since $p_{1}$ is not fixed by the $p_{2}-L_{2}$ perspectivities, there are other points $p$ on $L_{1}$ which are centers of $p-L_{1}$ transitivities. It follows from Theorem 7 that the plane is a Veblen-Wedderburn plane with $L_{1}$ as $L_{\infty}$. If we take $p_{2} \equiv B$ and $L_{2} \equiv(x=0)$, multiplication is associative by Theorem 6.

THEOREM 12. If $p_{1}$ is on $L_{1}$, neither $p_{1}$ nor $p_{2}$ is on $L_{2}$, and $p_{2}$ is not on $L_{2}$, then the plane is Desarguesian.

Proof. By the $p_{1}-L_{1}$ perspectivities, $p_{2}$ can be carried into any point on the line $p_{1} p_{2}$ except $p_{1}$. By the $p_{2}-L_{2}$ perspectivities, $p_{1}$ can be carried into any point on $p_{1} p_{2}$, excepting $p_{2}$ and the point on $L_{2}$. It follows that the group of collineations is transitive on points of $p_{1} p_{2}$ and every point on $p_{1} p_{2}$ is the center of two $p-L$ transitive groups. One of these is a group of 
elations and the other is a group of homologies. If we take any point on $p_{1} p_{2}$ as the point $p$ in Theorem 9 , we see that we have a nearfield plane. If $r$ is the intersection of $L_{1}$ and $L_{2}$, the line $p r$ will be our line at infinity. The existence of collineations moving the line at infinity implies the other distributive law (1, Theorem 18).

THeORem 13. If $L_{1}=L_{2}=L$ and neither $p_{1}$ nor $p_{2}$ is on $L$, the plane can be coordinatized by a dual nearfield.

Proof. Any point on the line $p_{1} p_{2}$ (excepting the point on $L$ ) can be carried into any other point. André (2, Theorem 3) has shown that the group of elations with axis $L$ is transitive on these points. That is, if $q$ is the intersection of $p_{1} p_{2}$ with $L$, the plane is $q-L$ transitive. Our theorem then follows from the dual of Theorem 9 .

THEOREM 14. If $p_{1}$ is on $L_{2}$ and $p_{2}$ is on $L_{1}$, but $p_{1}$ is not on $L_{1}$ nor $p_{2}$ on $L_{2}$, then the plane can be coordinatized by a system in which

(i) the coordinatization is linear,

(ii) multiplication is associative,

(iii) $a(c+b)=a c+a b$.

Proof. Take $L_{1}$ as $L_{\infty}, p_{1}$ as $(0,0), p_{2}$ as $B$ and $L_{2}$ as $x=0$. Then (i) and (ii) are established by Theorem 6 . Now, consider the $p_{1}-L_{1}$ perspeativity which carries the line $x=1$ into $x=a$. Since $(0,0)$ is the center, each line $y=x m$ is fixed, so that $(1, m) \rightarrow(a, a m)$. Thus, the line $y=m$ maps into $y=a m$. But, since the line $y=x$ is fixed, $(m, m) \rightarrow(a m, a m)$ and the line $x=m \rightarrow x=a m$. Now

$$
y=x+b \rightarrow y=x+a b .
$$

Therefore $(c, c+b) \rightarrow(a c, a c+a b)$. But $y=c+b \rightarrow y=a(c+b)$. Hence $a(c+b)=a c+a b$.

Theorem 15. If $p_{1}, p_{2}, p_{3}$ are the vertices of a triangle in which $L_{1}, L_{2}, L_{3}$ are the respective opposite sides and if the plane is $p-L$ transitive with respect to each vertex and its opposite side, the plane can be coordinatized by a neofield, i.e.,

$$
\begin{aligned}
& \text { (i) } x \cdot m \circ b=x m+b, \\
& \text { (ii) multiplication is associative, } \\
& \text { (iii) } a(c+b)=a c+a b \\
& \text { (iv) }(b+c) a=b a+c a .
\end{aligned}
$$

Proof. Take $p_{1}$ as $(0,0), p_{2}$ as $B, p_{3}$ as $A$. By Theorem 14 , we have only to prove (iv). In the group of perspectivities with center $A$ and axis $y=0$, consider the perspectivity which carries $y=1$ into $y=a$. Every line $x=c$ is fixed, so $(c, 1) \rightarrow(c, a)$. Now, $(0,0)$ is fixed, so $y=x c \rightarrow y=x m$ for some $m$. The point $(c, 1)$ is on $y=x c^{-1}$, so $(c, a)$ is on $y=x m$. Thus $a=c m$ and $m=c^{-1} a$ so that $\left(c^{-1}\right) \rightarrow\left(c^{-1} a\right)$. 
In particular, consider $y=x+c$, which goes through the point (1) and the fixed point $(-c, 0)$. Its image must go through $(a)$ and $(-c, 0)$. Thus

$$
y=x+c \rightarrow y=x a+c a .
$$

Therefore $(b, b+c) \rightarrow(b, b a+c a)$. But $y=b+c \rightarrow y=(b+c) a$. Hence $b a+c a=(b+c) a$.

We note here that Hughes (6) has used different groups of collineations to obtain coordinatinizations like those in Theorems 10,14 , and 15 .

THEOREM 16. If $p_{1}$ is not on $L_{1}$ and $p_{2}$ is not on $L_{2}$ and either (i) $p_{2}$ is on $L_{1}$ but $p_{1}$ is not on $L_{2}$ or (ii) $p_{1}$ is not on $L_{2}, p_{2}$ is not on $L_{1}$ and $p_{1}$ and $p_{2}$ are not collinear with the intersection $r$ of $L_{1}$ with $L_{2}$, then the plane is Desarguesian.

Proof. (i) Note that $L_{1}$ is fixed by both the $p_{1}-L_{1}$ perspectivities and the $p_{2}-L_{2}$ perspectivities. The points on $p_{1} p_{2}$ (excepting $p_{2}$ ) are transitive under collineations which leave $L_{1}$ fixed. Hence each point $p \neq p_{2}$ on $p_{1} p_{2}$ is the center of a $p-L_{1}$ transitive group. This implies (2, Theorem 3 ) that the plane is $p_{1}-L_{2}$ transitive.

By Theorem 9 , if we take $p_{2} \equiv A, L_{1} \equiv L_{\infty}, L_{2} \equiv(y=0)$ the plane will be coordinatized by a nearfield. If we take the line $p_{1} p_{2}$ as $x=0$, the plane will be $(0,0)-L_{\infty}$ transitive. By methods similar to those which have been used in other proofs, it can be shown that the $(0,0)-L_{\infty}$ perspectivity which carries $(1,1)$ into $(a, a)$ carries $y=c$ into $y=a c$ and $x=c$ into $x=a c$ and finally, that $a(c+b)=a c+a b$. Hence, the coordinatizing algebra is a field.

(ii) If neither $p_{1}$ nor $p_{2}$ is on $L_{1}$ or $L_{2}$ and $r$ is the intersection of $L_{1}$ and $L_{2}$, the $p_{1}-L_{1}$ and $p_{2}-L_{2}$ perspectivities generate a group which is doubly transitive on the points of $p_{1} p_{2}$ and leaves $r$ fixed. Hence, for every pair of points $q$ and $s$ on $p_{1} p_{2}(q \neq s)$, the plane is $q-s r$ transitive. Hence, keeping $s$ fixed and varying $q$, the plane is $s-s r$ transitive for each $s$ on $p_{1} p_{2}$ (2, Theorem 3). Taking $s$ as the point $p$ in Theorem 9 , the plane can be coordinatized by a nearfield with $s r$ as $L_{\infty}$. But $s r$ is not fixed, so the nearfield must be a field.

The above theorems characterize the various types of planes uniquely in the sense that any plane coordinatized by the algebra appropriate to each theorem will have the corresponding $p-L$ transitivities. If we include the cases dual to those considered here, we have taken care of all cases of pairs of $p-L$ transitivities except the following two:

I. Neither $p_{1}$ nor $p_{2}$ is on $L_{1}$ or $L_{2}$ but the line $p_{1} p_{2}$ goes through the intersection of $L_{1}$ and $L_{2}$.

II. $p_{1}$ is on $L_{1}$ and $p_{2}$ is on $L_{2}$ with $p_{1} \neq p_{2}$ and $L_{1} \neq L_{2}$.

\section{Multiple transitivity.}

Theorem 17. Let $\pi$ be a finite projective plane with $n+1$ points on a line. Then if $n$ is an odd power of two and the plane is doubly transitive, $\pi$ is Desarguesian. 
Proof. If $\pi$ is doubly transitive and not of square order, there is a perspectivity of period two (involution) with some center $p$ and axis $L$, where $p \in L$ for $n$ even (7). If $q \notin L$ and $q_{1} \neq L$ the existence of a collineation which fixes $p$ and carries $q$ into $q_{1}$ implies the existence of an involution with center $p$, axis $p q_{1}$. Thus every line through $p$ is an axis of a perspectivity of period two with center $p$. Now, by Lemma 2 , the product of two elations with the same center $p$ is an elation with center $p$. By Theorem 3 , all elations with the same center are of the same period. Hence the product of two involutions with center $p$ is an involution with center $p$, all axes going through $p$, since $n$ is even.

Thus the involutions with center $p$ form a group whose order is a power of 2 . The involutions with center $p$ and any given axis through $p$ form a subgroup. Let $2^{r}$ be the order of the group with center $p, 2^{s}$ the order of the group with center $p$, any given axis. Then, since there are $n+1$ lines through $p$,

$$
(n+1)\left(2^{s}-1\right)+1=2^{r}, 2^{s} n+2^{s}-n=2^{r},
$$

where $r>s$. Thus $2^{s}$ must divide $n$. Suppose $n=2^{s+t}$. Then

$$
2^{2 s+t}+2^{s}-2^{t+s}=2^{r}, 2^{s+t}+1-2^{\iota}=2^{r-s} .
$$

Now, since $r>s$, the right-hand side is even and the left-hand side is odd unless $t=0$. If such be the case, $n=2^{s}$. Thus the order of the group of involutions with center $p$, fixed axis through $p$, is equal to $n$. Hence we have $p-L$ transitivity for every line $L$ through $p$. This is true for every point in the plane. This implies projective satisfaction of the Minor Theorem of Desargues. Since the plane is finite, it must then be Desarguesian.

THEOREM 18. If $n$ is an odd power of two, and the plane is doubly transitive for points not on the line $L_{\infty}$, it is a Veblen-Wedderburn plane.

Proof. Unless $L_{\infty}$ is fixed by all collineations, the plane is doubly transitive for all points and we can apply Theorem 17. Suppose $L_{\infty}$ is fixed by every collineation of the plane. Now the double transitivity implies the existence of perspectivities of period 2. If $L_{\infty}$ is the axis of one of these involutions, any two points can be interchanged by an involution with $L_{\infty}$ as axis. Thus finite points are transitive under elations with $L_{\infty}$ as axis ("translations"), and the plane is Veblen-Wedderburn (1).

If $L_{\infty}$ is not the axis of any of these involutions, their centers must be on $L_{\infty}$ if $L_{\infty}$ is to be fixed by all collineations. The plane will be transitive on finite lines, so every finite line is the axis of an involution. Let $p$ be any point on $L$ and let $q$ be any finite point. Each involution which interchanges $q$ with a point on the line $p q$ must have $p$ as center. (The double transitivity implies that every pair of finite points can be interchanged by a perspectivity of order two.) Moreover, $p$ is the center of the involution with axis $p q$, since our involutions are elations. Corresponding to the $n$ finite lines through $p$, there are at least $n$ involutions with center $p$. 
Now, let $r \neq p$ be some point on $L_{\infty}$. Since $r$ has only $n$ possible images (including itself) under involutions with center $p$, either $r$ is fixed by some involution with center $p$ or at least two of them interchange $r$ with the same point. We are dealing with elations, so that the only fixed points are on the axes. Thus if $r$ is fixed by some involution, $L_{\infty}$ is the axis of that involution. We have already noted that the product of two involutions with center $p$ is an involution with center $p$. In the case where two involutions interchange $r$ with the same point, the product of these two involutions is an involution fixing $r$. In every case, we are led to an involution with $L_{\infty}$ as axis. We have already remarked that this is sufficient to establish the theorem.

Now, every collineation of period two either is a perspectivity or leaves fixed all of the points of a subplane of order $n^{\frac{1}{2}}$. The author's proof that doubly transitive planes are Desarguesian fails only in the case that no collineation of period two is perspectivity. Thus (even if $n$ is a square) if $n$ is odd or a power of two and the plane is doubly transitive, it is Desarguesian unless every collineation of period two leaves a subplane of order $n^{\frac{1}{2}}$ pointwise fixed.

Definition. A plane which contains no proper subplanes will be called a minimal plane.

THEOREM 19. If the plane $\pi$ is finite and is transitive on proper quadrangles, then every quadrangle generates a Desarguesian subplane of prime order $\mu$, where $\mu$ divides $n$ or $n-1$.

Proof. If $\pi$ is finite, it certainly contains minimal subplanes. The key to our argument is the fact that two minimal subplanes cannot share a quadrangle. Let $\pi_{2}$ be a minimal subplane of $\pi$. Any collineation of $\pi$ which carries a quadrangle of $\pi_{1}$ into a quadrangle of $\pi_{2}$ is also a collineation of $\pi_{1}$. Hence $\pi_{1}$ is transitive on quadrangles and is doubly transitive. If the order of $\pi_{1}$ is odd, $\pi_{1}$ is Desarguesian. If the order $\mu$ of $\pi_{1}$ is even, $\pi_{1}$ will be Desarguesian if $\mu$ is a power of 2. Now $\pi_{1}$ admits perspectivities of period two. But if $\mu$ is even this implies that $\pi_{2}$ contains a Fano subplane (8). Thus if $\mu$ is even, $\mu$ is equal to 2 .

Clearly, every quadrangle will generate a subplane of order $\mu$ and since $\pi_{1}$ is a minimal Desarguesian plane, $\mu$ is a prime.

Now consider three fixed points $p, q$, and $r$ on a line $L$ and a line $L_{1} \neq L$ through $p$. Let $s \neq p$ be a fixed point on $L_{1}$. There will be $n-1$ quadrangles such that $q$ and $r$ are diagonal points, $s$ is one vertex and $L_{1}$ is one side. The points $p, q$, and $r$ will be in the minimal subplane generated by any one of these quadrangles. These quadrangles will occur in subsets with $\mu-1$ in each subset, those in the same subset belonging to the same minimal subplane. Hence $\mu-1$ divides $n-1$. Similarly, if we allow $s$ to vary, $\frac{1}{2} \mu(\mu-1)$ divides $\frac{1}{2} n(n-1)$. Since $\mu$ is prime, $\mu$ divides $n$ or $n-1$.

Furthermore, there are $\left(n^{2}+n+1\right)\left(n^{2}+n\right) n^{2}(n-1)^{2}$ ordered quadrangles in $\pi$ and

$$
\left(\mu^{2}+\mu+1\right)\left(\mu^{2}+\mu\right) \mu^{2}(\mu-1)^{2}
$$


ordered quadrangles in each minimal subplane. Hence

$$
\left(\mu^{2}+\mu+1\right)\left(\mu^{2}+\mu\right) \mu^{2}(\mu-1)^{2}
$$

divides $\left(n^{2}+n+1\right)\left(n^{2}+n\right) n^{2}(n-1)^{2}$. If we consider the class of quadrangles containing some fixed point, we get by a similar argument that $\left(\mu^{2}+\mu\right) \mu^{2}(\mu-1)^{2}$ divides $\left(n^{2}+n\right) n^{2}(n-1)^{2}$.

\section{The existence of Desarguesian configurations.}

Theorem 20. In a finite projective plane, let $L_{1}, L_{2}$ and $L_{3}$ be three distinct lines through a point p. Let $r$ and $s$ be any two points not on $L_{1}, L_{2}$ or $L_{3}$. Consider the set of triangles with one vertex each on $L_{1}, L_{2}$, and $L_{3}$, one side going through $r$ and the other side going through s. At least one pair of triangles of this set satisfies Desargues's Theorem.

Proof. Let $L_{1}, L_{2}$, and $L_{3}$ respectively intersect the line $r s$ in the points $t_{1}, t_{2}$, and $t_{3}$. Let $A_{1}, A_{2}$, and $A_{3}$ be the vertices of a triangle such that $A_{1}$ is on $L_{1}, A_{2}$ is on $L_{2}$, and $A_{3}$ is on $L_{3}$. Let these points be chosen so that the line $A_{2} A_{3}$ goes through $r$ and $A_{2} A_{1}$ goes through s. $A_{2}$ may be any point on $L_{2}$ except $p$ or $t_{2}$. Hence there are $n-1$ such triangles.

Now the line $A_{1} A_{3}$ cannot intersect $r s$ in $t_{3}$ or $t_{2}$. Neither can $A_{1} A_{3}$ go through $r$ or $s$. Hence there are at most $(n+1)-4=n-3$ possible points in which $A_{1} A_{3}$ can intersect $r s$. Since there are $n-1$ such triangles, there must be a pair of triangles $A_{1} A_{2} A_{3}$ and $A_{1}{ }^{\prime} A_{2}{ }^{\prime} A_{3}{ }^{\prime}$ such that $A_{1} A_{3}$ and $A_{1}{ }^{\prime} A_{3}{ }^{\prime}$ intersect in a point on $r s$. The triangles $A_{1} A_{2} A_{3}$ and $A_{1}{ }^{\prime} A_{2}{ }^{\prime} A_{3}{ }^{\prime}$ then satisfy Desargues's Theorem.

A similar theorem can be proved for $r=t_{3} s=t_{1}$ if Fano's configuration does not exist in the plane.

Added in proof. Since submitting this paper, the author has received communications from A. Wagner which make Theorems 17 and 19 outmoded. Wagner has shown that (1) double transitivity implies that the plane is Desarguesian provided only that $n$ is even, and (2) without regard to restrictions on $n$, transitivity on quadrangles implies that the plane is Desarguesian. 


\section{REFERENCES}

1. J. André, Ueber nicht-Desarguesche Ebene mit transitiver Translationsgruppe, Math. Z., 60 (1954), 156-186.

2. - Ueber Perspektivitäten in endlichen projektiven Ebenen, Arch. Math., 6 (1955), 29-32.

3. - Projektive Ebenen über Fastkörpern, Math. Z., 62 (1955), 137-160.

4. R. Baer, Homogeneity of projective planes, Amer. J. Math., 64 (1942), 137-152.

5. M. Hall, Projective planes, Trans. Amer. Math. Soc., 54 (1943), 229-277.

6. D. R. Hughes, Partial difference sets, Amer. J. Math., 78 (1956), 650-674.

7. T. G. Ostrom, Double transitivity in finite projective planes, Can. J. Math., 8 (1956), 563567.

8. - Ovals, dualities, and Desargues's theorem, Can. J. Math., 7 (1955), 417-431.

9. G. Pickert, Projektive Ebenen (Berlin, 1955).

Montana State University 